\title{
II. - UTILISATION DIGESTIVE ET VALEUR NUTRITIVE DE POMMES A L'ÉTAT FRAIS
}

PAR

\author{
G. CHARLET-LERY, A. M. LEROY, S. Z. ZELTER
}

Les quelques rares essais d'alimentation avec pommes fraîches, qui ont été effectués sur vaches laitières, moutons et pores (I) ne fournissent pas de données relatives aux coefficients de digestibilité des divers constituants de ce fruit soit à l'état frais, soit après ensilage.

La recherche des possibilités d'écoulement des fruits à cidre en excédent nous a conduits à déterminer l'utilisation digestive d'un mélange de pommes en nature.

Les ruminants consommant, soit au verger, soit à l'auge, des quantités importantes de pommes à cidre fraiches, nous avons expérimenté avec des moutons afin de connaitre la valeur nutritive de cet aliment.

\section{Technique expérimentale.}

Notre technique de mesure de la digestibilité ayant été décrite dans d'autres mémoires (2-3), nous précisons qu'en l'occurrence la durée de chacune des périodes expérimentales et des périodes d'adaptation précédant chaque expérience est d'au moins ro jours.

I Les 4 sujets adultes, femelles, de race Ile-de-Iirance utilisés pèsent de 49 à $56 \mathrm{~kg}$ et sont exempts de parasites intestinaux.

L'étude est effectuée avec le mélange de 3 variétés de pommes, qui a servi à des recherches sur la conservation (voir mémoire I). La teneur de ces pommes en matière sèche (moyenne de 20 déterminations en tunnel infra-rouge) a oscillé au cours de l'expérience entre I8,8 et I9 p. Ioo; les sucres solubles représentent 47,6 et les glucides hydrolysables $56,8 \mathrm{p}$. Ioo de cette matière sèche. Les fruits sont incorporés dans une ration mixte dont l'aliment de base est un foin de luzerne; suivant les périodes :

o - 30,5 - 34,9 ou 45,3 p. Ioo de la matière sèche totale ingérée sont fournis par les pommes.

Ces pourcentages - même les plus élevés — ne modifient pas l'appétence.

La composition des constituants de chacune des rations se trouve 
dans le tableau I. Les niveaux de consommation journalière de matière sèche varient selon les sujets et les périodes entre I 2 I7 et I $328 \mathrm{~g}$.

\section{TABleaU I}

Composition des aliments en $p$. I ooo de matière sèche.

\begin{tabular}{|c|c|c|c|c|c|c|}
\hline & $\begin{array}{c}\text { Matière } \\
\text { Minérale }\end{array}$ & $\begin{array}{c}\text { Matières } \\
\text { Organiques }\end{array}$ & Azote & $\begin{array}{c}\text { Matière } \\
\text { Grasse }\end{array}$ & $\begin{array}{l}\text { Cellulose } \\
\text { Weende }\end{array}$ & $\begin{array}{l}\text { Extractifs } \\
\text { non azotés }\end{array}$ \\
\hline $\begin{array}{c}\text { Régime à o } \% \text { de pomme } \\
\text { Foin } \ldots \ldots \ldots \ldots \ldots \ldots \ldots \\
\text { Régime à } 30,5 \% \text { de matière } \\
\text { sèche de pomme: }\end{array}$ & 104,5 & 895,5 & $3 \mathrm{r}, \mathrm{I}$ & $4^{6,9}$ & 248,6 & $4 \mathrm{I} 3,4$ \\
\hline Foin $\ldots \ldots \ldots \ldots$ & $98, I$ & 901,9 & 30,6 & 32,6 & 238,5 & 447,6 \\
\hline $\begin{array}{l}\text { Pomme } \ldots \ldots \ldots \ldots \ldots \\
\text { Régime à } 34,9 \% \text { de matière } \\
\text { sèche de pomme : }\end{array}$ & $20, \mathrm{I}$ & 979,9 & 3,2 & 6,9 & 67,7 & 885,9 \\
\hline Foin .................... & $9^{6,3}$ & 903,7 & $3 \mathrm{I}, 2$ & 37,9 & 254,8 & 423,7 \\
\hline $\begin{array}{l}\text { Pomme } \ldots \ldots \ldots \ldots \ldots \\
\text { Régime à } 45,35 \% \text { de ma- } \\
\text { tière sêche de }\end{array}$ & 22,9 & $977, \mathrm{I}$ & 5,0 & 8,6 & $7 \mathrm{I}, 3$ & 867,4 \\
\hline 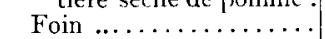 & 95,4 & $9 c_{4}, 6$ & 29,3 & 39,8 & 255,8 & 433,2 \\
\hline Pomme ............. & 21,8 & 978,2 & 4,0 & I 5,8 & 76,5 & 861,6 \\
\hline
\end{tabular}

\section{RÉSULTATS}

Le tableau II donne les coefficients moyens de digestibilité des éléments des régimes mixtes. Les données individuelles n'y figurent pas, les écarts observés entre sujets étant très faibles ( 2 I $/ 2$ points au maximum pour la matière organique).

\section{TABLEAU II}

Coefficients moyens d'utilisation digestive des divers constituants des régimes.

\begin{tabular}{|c|c|c|c|c|}
\hline & $\begin{array}{c}\text { Expérience I } \\
\text { (Régime à } \\
30,5 \% \text { de } \\
\text { pommes) }\end{array}$ & $\begin{array}{c}\text { Expérience II } \\
\text { (Régime à } \\
45,35 \% \text { de } \\
\text { ponmes) }\end{array}$ & \begin{tabular}{|} 
Expérience III \\
(Régime à \\
$34,9 \%$ de \\
pommes)
\end{tabular} & $\begin{array}{l}\text { Période Témoin } \\
\text { (Régime à } \\
\text { I00\% de foin) }\end{array}$ \\
\hline $\begin{array}{l}\text { Matière sèche } \ldots \ldots \ldots \ldots \ldots \ldots \\
\text { Matières organiques } \ldots \ldots \ldots \ldots \ldots \\
\text { Azote } \ldots \ldots \ldots \ldots \ldots \ldots \\
\text { Matière grasse } \ldots \ldots \ldots \ldots \ldots \ldots \\
\text { Cellulose } \ldots \ldots \ldots \ldots \ldots \ldots \\
\text { Extractifs non azotés } \ldots \ldots \ldots \ldots\end{array}$ & $\begin{array}{l}69,4 \\
72,7 \\
68,6 \\
11,6 \\
46,0 \\
84,8\end{array}$ & $\begin{array}{l}74,5 \\
77,0 \\
63,9 \\
20,4 \\
51,1 \\
88,3\end{array}$ & $\begin{array}{r}70,2 \\
74,2 \\
66,0 \\
9,9 \\
54,0 \\
85,2\end{array}$ & $\begin{array}{l}6 \mathrm{I}, 2 \\
64,2 \\
76,2 \\
49,2 \\
4 \mathrm{I}, 2 \\
74,7\end{array}$ \\
\hline
\end{tabular}

L,e tableau III contient les coefficients individuels, calculés par différence, des divers principes nutritifs des pommes fraîches, en fonction de leur taux d'incorporation dans le régime. 


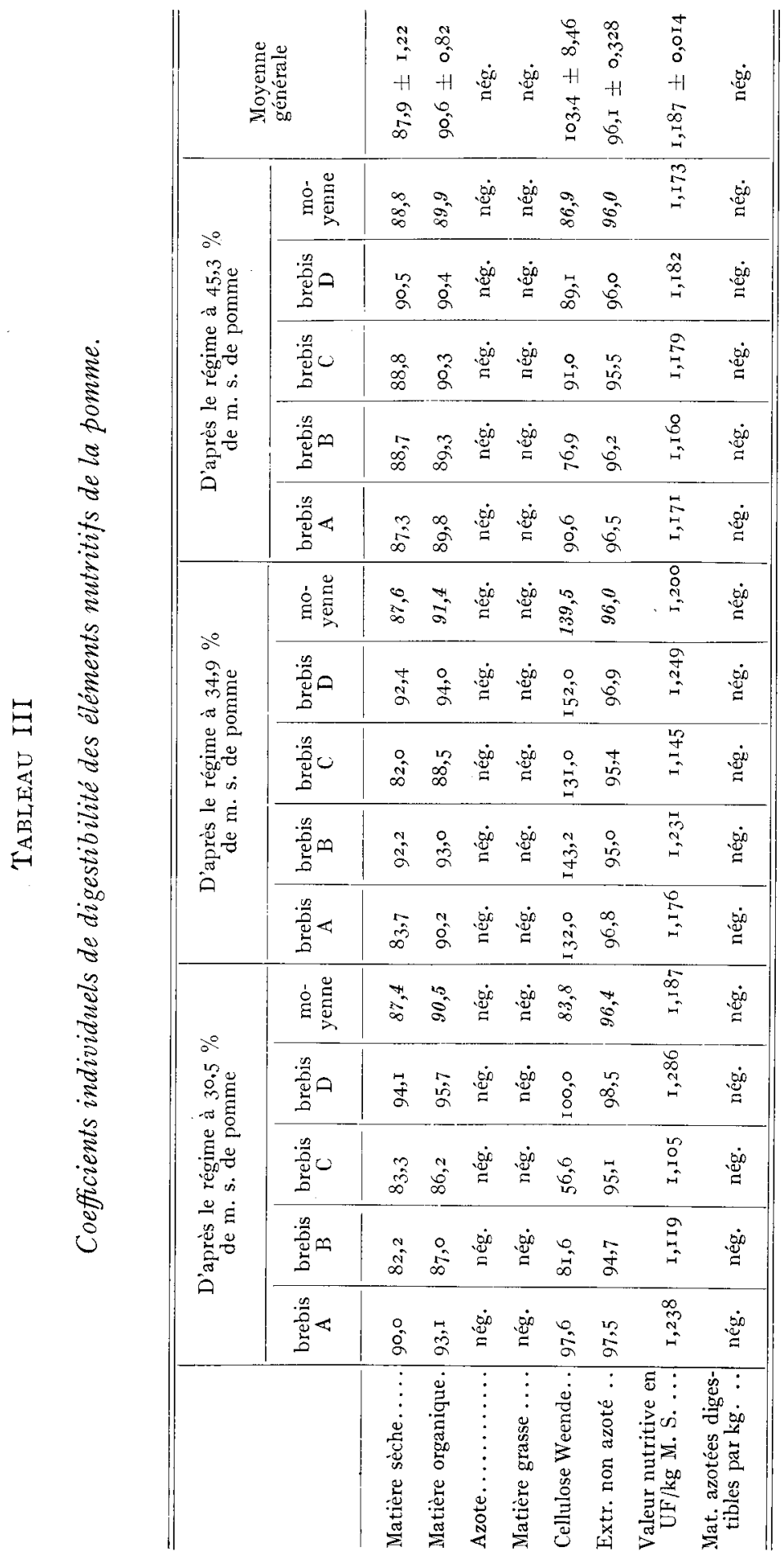




\section{DISCUSSION}

I1 est intéressant de souligner la parfaite homogénéité des résultats observés chez le mouton.

I,es données du tableau III font ressortir pour la pomme que :

-- le pourcentage de matière sèche de ce fruit dans le régime oscillant entre 30 et 45 n'exerce pas d'influence sur le taux d'utilisation digestive de ses divers constituants;

- la digestibilité de la matière sèche, de la matière organique et des extractifs non azotés est très élevée. Les valeurs moyennes respectives sont : 87,9 p. Ioo - 90,6 p. Ioo et 96, I p. Ioo ;

- la digestibilité de la cellulose Weende calculée à partir des régimes comportant 30,5 et 45,3 p. Ioo de matière sèche de pomme, est exceptionnellement élevée : 83,8 et 86,9 p. Ioo. Elle peut s'expliquer par le fait que la majeure partie de la cellulose brute s'y trouve à l'état de cellulose vraie $(84,2$ p. Ioo de cellulose Kurschner). Il se pourrait que la très faible part de cellulose brute de la ration en provenance de la pomme ait entraîné un résultat aberrant (I39,5 p. IOO) dans le cas du régime à 34,9 p. IOO ;

- l'utilisation digestive des fractions azotée et lipidique est négative quel que soit le pourcentage de pomme consommé; un fait analogue a déjà été rapporté pour le premier de ces éléments dans le cas de l'alimentation avec du marc de pomme (5);

- la valeur nutritive de la matière sèche de pomme est très forte ; elle atteint en moyenne I,I87 $\pm 0,0 \mathrm{I} 4 \mathrm{u}$. f. $/ \mathrm{kg}$. Ce qui correspond à un équivalent fourrager de $4,45 \mathrm{~kg}$ pour le mélange de variétés expérimentées qui contient I9 p. Ioo de substance sèche.

\section{RÉSUMÉ}

Une étude d'utilisation digestive d'un mélange de pommes à cidre a été faite sur le mouton à l'état frais.

I es résultats montrent que, chez ce ruminant les digestibilités des matières sèche, organique et glucidique de la pomme fraîche sont extrêmement élevées, d'où une valeur nutritive de I,I $87 \pm$ o,oI4 unité fourragère par $\mathrm{kg}$ de matière sèche.

I a digestibilité des fractions azotée et lipidique est négative.

\section{RÉFÉRENCES BIBLIOGRAPHIQUES}

(I) Morrisson (F. B.). - Feeds and Feeding, I950, p. 457 (Ithaca-NewYork).

(2) Leroy (A. M.) Lery (G.). - Ann. Agron., I947, no 2, p. 264.

(3) Leroy (A. M.) Lery (G.), Zei.Ter (S. Z.). - Ann. Zoot., 1, no I, p. 29, I952. 